\title{
Refeeding Syndrome
}

\section{Emel KOÇER, Aynur GÖRMEZ}

Department of Psychiatry, Bezmialem Vakıf University School of Medicine, İstanbul, Turkey

\section{ABSTRACT}

Refeeding syndrome (RFS) can be defined as a condition with severe electrolyte disturbance and metabolic abnormalities in undernourished patients who are undergoing refeeding. It is a life-threatening condition if not recognized or managed properly. Although there are no universally agreed guidelines on managing RFS, being aware of high-risk groups with close monitoring and a multidisciplinary approach is crucial for prevention as well as management. It is of paramount importance that clinicians are made more aware of this preventable, yet fatal, syndrome. There is also a great need for more randomized controlled trials to be performed, which can guide the clinical practice.

Keywords: Refeeding syndrome, malnutrition, hypophosphatemia

\section{Introduction}

Refeeding syndrome (RFS) is a dangerous consequence of a series of metabolic and biochemical changes that occur as a result of excessive and rapid food intake in people with severe malnutrition. It was first observed after the Second World War when the prisoners of war regained their freedom and started eating again after long periods of poor nutrition and living under deprived conditions, which led to sudden death. These individuals developed a number of problems that were initially believed to be caused by a variety of gastrointestinal (GI) factors. However, no basic GI pathology could be detected in the autopsies. It was later reported that the phenomenon was associated not only with poor living conditions but also with many medical reasons. It was commonly observed with the enteral or parenteral refeeding of patients, particularly in those who lost $10 \%$ of their body weight in the last few months (1).

\section{Clinical Features}

Particularly with the prolongation of the duration, the clinical appearance of RFS can be quite different. Because it has a large clinical spectrum, it may cause serious consequences for patients in the risk group if its presence is not taken into consideration or is disregarded.

The following patient groups are at risk for RFS (2):

- Patients chronically malnourished due to cancer, irritable bowel syndrome, bowel fistulas, and old age

- Patients suffering from long-term hunger (due to war, captivity, famine, and the death fast)

- Patients suffering from conditions that disrupt food intake, such as anorexia nervosa (AN), chronic alcoholism, and severe depression

- Obese patients with apparent and rapid weight loss, particularly after duodenal switch surgery

- Malnourished infants (kwashiorkor and marasmus syndromes)

Cite this article as: Koçer E, Görmez A. Refeeding Syndrome. Bezmialem Science 2017; 5; 121-5. 
Data containing relative incidences of clinical manifestations are inadequate. The incidence of RFS was found to be as high as $25 \%$ in cancer patients receiving total nutritional support (1). In the study, the incidence has been reported to be higher in enteral feeding than in parenteral feeding. The condition tends to become evident in the first few days of feeding. It is more common in elderly people and poses a serious risk in terms of mortality due to accompanying underlying diseases. In a study evaluating feeding during the rehabilitation of patients with AN, moderate hypophosphatemia was detected in one-fourth of the patients before a clinical picture emerged (3). Although the syndrome is known, no prospective study related to its incidence has been found so far, and our knowledge is based mostly on cases, case series, and retrospective evaluations. Electrolyte disorders that occur primarily in the first few days of refeeding cause cardiac problems, delirium, and other neurological symptoms within the next few weeks. Although there is a tendency to perceive it as a deficiency of electrolytes, vitamins, and nutrients in general, it actually involves much more complex processes. RFS is the result of a series of metabolic and biochemical changes. The RFS symptoms are variable and unpredictable, and they may emerge insidiously and too late (4). Mild irregularities in the levels of electrolytes may not be symptomatic. RFS frequently begins with nasal discharge, vomiting, and lethargy and extends to hypotension, arrhythmia, respiratory failure, heart failure, coma, delirium, and death. While it progresses slowly in the beginning, the clinical picture may settle quickly.

To understand the clinical manifestations that occur in RFS, first, it is necessary to know the basic physiological changes that occur in the body due to hunger. In general, carbohydrates taken up with nutrition provide most of our energy needs. Glucose, the primary product of carbohydrate digestion, is actively transported along with sodium $(\mathrm{Na})$ from the intestinal epithelium across the concentration gradient. Glucose is absorbed through diffusion and enters the portal circulation, and consequently, the blood sugar levels rise. This stimulates the release of the insulin hormone from the pancreas. Insulin has many effects: It supports glucose uptake and storage, prevents the breakdown of fat cells (lipolysis), and increases potassium intracellular uptake. When the glycogen storage capacity of the liver cells is exceeded, non-oxidized glucose is converted into fat and stored as triglyceride in fat tissue. The explicit result of this is a decrease in blood glucose levels.

Glucose levels begin to decrease within 24-72 hours in individuals who do not receive nutrients due to famine or hunger. This results in a decreased insulin secretion and the release of the glucagon hormone. During hunger, glucagon concentrations increase, while insulin concentrations decrease. Glucose levels are maintained by glycogenolysis, but glycogen stores are rarely sufficient for more than 72 hours. Glucose homeostasis is required for certain tissues that are obligate glucose users, such as the brain, erythrocytes, and renal medullary cells. This need triggers glyconeogenesis, which causes glucose synthesis from lipids and protein degradation products, and at the same time, causes a rapid transformation of glycogen, which is the storage form of glucose. While large quantities of free fatty acids (FFAs) and glycerols are released from the adipose tissue, amino acids are also released from the muscle tissue into the blood stream. In fact, all of this is an effort to compensate the glucose that the body cannot get from external food resources through internal resources. Under these conditions, ketone bodies and FFAs take the place of glucose as the main energy source. In general, the catabolism occurring in muscle and fat tissue results in a loss in the body mass index. Loss of fat and protein in the body is accompanied by potassium, phosphate, and magnesium depletion. Homeostatic mechanisms maintain the serum concentrations of these ions at the expense of using intracellular stores. For this reason, although there is a noticeable decrease in total body levels, the serum levels of these electrolytes may remain normal for at least a while. With the resumption of nutrient intake, a shift from fat to carbohydrate metabolism is observed. The rapidly taken glucose load stimulates the release of insulin and leads to an increase in protein synthesis and the uptake of water, magnesium, potassium, phosphate, and glucose. This leads to a rapid fall in the extracellular compartment. $\mathrm{Na}$ and water retention try to maintain osmotic neutrality. The reactivation of carbohydrate-dependent metabolic pathways increases the need for thiamine, which is the cofactor for many enzymatic reactions. Different effects occur in different patients with varying levels of phosphate, magnesium, potassium, and thiamine. Some situations, such as long-term starvation or chronic alcohol dependence, make the individual more susceptible in terms of metabolic consequences.

One of the primary features of RFS is hypophosphatemia (1). Although low levels of phosphate (PO4) are not pathognomonic, they are accepted as markers for RFS diagnosis. PO4 is a major intracellular anion. In adults, $80 \%$ of the PO4 stores are in the skeleton and 20\% are in the soft tissues and muscles. The intracellular transport of $\mathrm{PO} 4$ is affected by acid-base changes and by lipid and carbohydrate digestion. For example, acidosis causes PO4 to exit the cell. The daily intake of PO4 is approximately $1 \mathrm{~g}$, and dietary PO4 deficiency is not common; in fact, it is usually consumed more than needed. Furthermore, $60 \%-70 \%$ is absorbed from the jejunum and $>90 \%$ is excreted through the kidneys. Most of the amount filtered from the glomeruli is reabsorbed from the proximal tubules, and this system is important for the control of the PO4 balance. GI losses correspond to $10 \%$ of the PO4 excretion. PO4 is necessary for many functions of the cell. It is an important intracellular buffer and is needed to buffer hydrogen ions in urine. PO4 is a structural element of phospholipids, nucleoproteins, and nucleic acids. In addition, it plays a central role in metabolic pathways, including glycolysis and oxidative phosphorylation. One of the products of glycolysis is 2,3-diphosphoglycerate, which is a regulator for the decomposi- 
tion of oxygen from hemoglobin $(\mathrm{Hb})$ and for the delivery of oxygen to the tissues. Eighty percent of the organic PO4 is found in erythrocytes. PO4 is involved in many enzymatic processes. An important control mechanism for the enzyme activation is the phosphorylation of proteins. Nucleotides such as adenosine triphosphate (ATP) contain phosphorus (P). Another role is the excitation-stimulus response and nervous system communication. $\mathrm{PO} 4$ has great importance in the optimal function of leukocytes, such as chemotaxis and phagocytosis, and in the coagulation reaction of platelets. When considered in light of all this general information, the abundance of clinical manifestations of heavy hypophosphatemia (plasma inorganic phosphate level below $0.30 \mathrm{mmol} / \mathrm{l}$ ) can be understood. In practice, severe hypophosphatemia is rare, and most cases progress asymptomatically. However, it is the result of the fact that the cell energy pathways, such as clinical ATP and 2,3-diphosphoglycerate, are affected in severe cases. Reduced skeletal muscle functions, weakness, and rhabdomyolysis associated with myopathies have been described. By affecting the diaphragm muscles, hypophosphatemia can lead to respiratory failure to such an extent that would require mechanical ventilation support. Cardiomyopathies are another important complication. Seizures, mental impairment, and paresthesia can be seen in the nervous system with the effect of hypophosphatemia. Unexpectedly prolonged hypophosphatemia can lead to osteomalacia. Renal tubular insufficiency is precipitated by hypophosphatemia, and acute tubular necrosis can lead to secondary rhabdomyolysis. Hematologic effects include thrombocytopenia, impaired coagulation processes, decreased leukocyte phagocytosis, and chemotaxis. Hemolysis can occur due to 2,3-diphosphoglycerate depletion, and this results in a left shift in the $\mathrm{Hb} / \mathrm{O} 2$ dissociation curve. In other words, the affinity of $\mathrm{Hb}$ to $\mathrm{O} 2$ further increases. To date, two fatal refeeding cases wherein hypophosphatemia was very severe and triggered by total parenteral nutrition have been reported $(5,6)$.

Another laboratory finding in RFS is hypomagnesemia (1). Magnesium $(\mathrm{Mg})$ is an abundant cation in the intracellular compartment, essential for optimal cell functions. $\mathrm{Mg}$ is required as a cofactor of many enzymes. It is mainly found in muscles and bones in the body. $\mathrm{Mg}$ is absorbed through the upper region of the small intestine. Up to $70 \%$ of the dietary $\mathrm{Mg}$ is excreted in feces without being absorbed. $\mathrm{Mg}$ is mainly excreted through the kidneys. The findings of RFS are also related to hypomagnesemia, but the mechanism is not clear. For example, an inadequate uptake of $\mathrm{Mg}$ through the diet may disrupt the transport of ions into the cells fed with carbohydrates. Cardiac arrhythmias, which also include Torsades de Pointes syndrome, may be seen in cases of severe hypomagnesemia (plasma concentration $<0.50 \mathrm{mmol} / \mathrm{l}$ ). Additionally, abdominal discomfort and anorexia have been defined. There may also be neuromuscular signs, such as tremor, paresthesia, tetany, seizures, irritability, confusion, and ataxia.
An additional expected electrolyte disorder is hypokalemia (1). Potassium $(\mathrm{K})$ is a cation that is necessary to maintain cell membrane action potentials. Total body potassium is regulated by the kidneys. The distal nephron secretes $\mathrm{K}$ into the urine; alkalosis, a high potassium diet, and elevated $\mathrm{Na}$ levels in the distal tubule affect $\mathrm{K}$ excretion by increasing aldosterone. Hypokalemia may lead to hypotension, cardiac arrhythmia, and cardiac arrest. It also triggers ileus and constipation in the GI system. It reduces the ability of the kidney to concentrate urine and causes neuromuscular dysfunctions, such as weakness, paralysis, paresthesia, confusion, and respiratory depression. In contrast, it potentiates digitalis toxicity and aggravates the situation in glucose tolerance, metabolic alkalosis, and hepatic encephalopathy. Hypokalemia has been defined as a plasma $\mathrm{K}$ level $<3.0 \mathrm{mmol} / \mathrm{l}$, and the clinical complications become apparent after this point. This situation is rarely seen without hypophosphatemia and hypomagnesemia.

In RFS, disturbances in body fluid distribution can affect many body functions (1). Fluid imbalance can lead to hypotension, heart failure, dehydration or fluid uptake, prerenal insufficiency, and sudden death. Carbohydrate-weighted refeeding can reduce water and $\mathrm{Na}$ excretion; in particular, if dietary $\mathrm{Na}$ uptake has increased, it causes expansion in the extracellular fluid compartment. Protein- and lipid-weighted refeeding can lead to weight loss and a negative $\mathrm{Na}$ balance, resulting in $\mathrm{Na}$ secretion from the urine. High-protein refeeding leads to hypernatremia associated with hypertonic dehydration, azotemia, and metabolic acidosis.

Abnormal glucose and lipid metabolism also occurs in RFS (1). Glycogenesis is suppressed due to a decrease in the lownegative nitrogen balance and in the use of amino acids along with glucose uptake. The first glucose infusion may subsequently cause hyperglycemia. Then, a hyperosmolar nonketotic coma can induce ketoacidosis, metabolic acidosis, osmotic diuresis, and dehydration. Turning into fat through lipogenesis, excess glucose can cause a fatty liver and abnormal liver function tests, as well as hypercapnia and respiratory failure with increased carbon dioxide production. Thus, daily fat production should not exceed the maximum fat elimination capacity, which is $3.8 \mathrm{~g} / \mathrm{kg}$.

Vitamin B1 (thiamine) deficiency is also associated with refeeding (7). Patients with malnutrition may have different vitamin deficiencies, but thiamine can be replaced through nutrition. Thiamine deficiency may cause Wernicke's encephalopathy and Korsakoff's psychosis. Ocular disorders are associated with confusion, ataxia, short-term memory loss, confabulation, and coma. The chronic use of alcohol facilitates the emergence of the mentioned clinical pictures. Refeeding with carbohydrate leads to an increase in the intracellular uptake of thiamine, which is a cofactor in various enzyme activities, such as transketolases. For this reason, the compensation of thiamine deficiency during refeeding can reduce the symptoms. 


\section{Management of RFS}

The basic principle in the prevention and treatment of RFS is identifying the patients at risk for RFS development and monitoring the nutritional intake and fluidelectrolyte replacement by an experienced multidisciplinary team. There are very different opinions regarding the speed and content of the ideal nutritional regimen, but there is little qualified evidence to support them in the literature. Nevertheless, in light of current clinical and follow-up findings, the management of RFS can be summarized as follows:

\section{1) Identification and Follow-Up of Patients in the High- Risk Group}

As mentioned above, patients who are at risk of RFS development can be listed as follows: patients with eating disorders, chronic alcoholism, substance abuse, advanced age, inadequate nutrition (e.g., kwashiorkor and marasmus), long-term hunger and vomiting, inflammatory bowel disease, malabsorption, short bowel syndrome, cancer, chronic infections (e.g., the human immunodeficiency virus); patients who underwent bariatric surgery; and infants with a low birth weight and those who are premature.

\section{2) Regulation of Problems in Fluid Balance}

Fluid intake should be sufficient to meet adequate hydration, and the patient's body weight should be monitored daily to prevent excess fluid uptake. A recent study has suggested that low $\mathrm{Na}$ intake reduces the risk of edema depending on refeeding (8). In the presence of hyponatremia, rapid replacement should be avoided due to the risk of central pontine myelinolysis. Renal functions should also be closely monitored because patients with RFS have a higher renal risk.

\section{3) Regulation of Electrolyte Deficiencies and Acid-Base Balance Disorders}

Instead of trying to correct the fluid intake and imbalance in electrolytes $(\mathrm{Mg}, \mathrm{K}$, and PO4) before the feeding, it is important to do it during the feeding in order not to delay the energy intake (2). PO4, Mg, and $\mathrm{K}$ levels should be measured daily until they return to normal, and low values should be supplemented without delay. In addition, $\mathrm{Na}$, bicarbonate, and chlorine levels should be measured for the acid-base balance. For example, due to thiamine deficiency, glucose metabolism may be impaired, and lactic acidosis may occur.

\section{4) Vitamin Supplements}

Many guides recommend vitamin supplements 10 days before and after refeeding is started. Thiamine should be given at a dose of $200-300 \mathrm{mg} /$ day, B vitamin complexes 3 times a day, and a multivitamin supplement once a day (2). Serum ferritin, vitamin B12, and folic acid levels should also be monitored.

\section{5) Monitoring Trace Elements}

Trace elements should be measured in the beginning and every 1-2 weeks; if there is a deficiency, zinc should be replaced at a dose of $2.5-5 \mathrm{mg} /$ day, selenium at a dose of $20-70 \mathrm{mg} /$ day, manganese at a dose of $3.5-7 \mathrm{mg} /$ day, and copper at a dose of $0.3-0.5 \mathrm{mg}(9)$.

\section{6) Energy Supplement}

Some recent studies have indicated that the possibility of RFS development increases when carbohydrates are given as the main energy source (10). The content of nutrition is more important than its total calorie count, and recent studies have suggested that carbohydrates should be given to a lesser extent, for example, in an amount that would constitute only $40 \%$ of the total energy. The usual daily protein requirement is approximately $1.2-1.5 \mathrm{~g} / \mathrm{kg}$. Fat intake should be at a lipid ratio of approximately $3.8 \mathrm{~g} / \mathrm{kg}$, so as not to exceed the daily lipid elimination capacity.

\section{7) Parameters Requiring Follow-Up}

Daily body weight, the follow-up of the intake/discharge ratio, fluid uptake, and associated complications are important to prevent cardiac insufficiency. Vital signs should be monitored daily; for example, tachycardia may be a sign of cardiac insufficiency. Plasma glucose should be measured daily to prevent hypo/hyperglycemia. Liver function tests should also be performed due to the risk of plasma albumin, protein, and calcium levels and due to the risk of hemogram and liver dysfunction.

There are recent publications that draw attention to aggressive nutrition and malnutrition dichotomy. An extremely prudent calorie intake can also lead to underfeeding syndrome, which can increase mortality and morbidity. MARSIPAN (Management of Really Sick Patients with Anorexia Nervosa) and a large number of studies conducted in patients with severe AN have presented findings suggesting that energy intakes as high as $40 \mathrm{kcal} / \mathrm{kg}$ do not result in RFS $(11,12)$. O'Connor and Nicholls pointed out that the amount of energy coming from glucose is important and suggested that the risk of RFS can theoretically be lowered by minimizing the rate of rapid insulin release by reducing the rate of glucose and increasing the rate of fat (3).

According to the Nutrition Guidelines for Adults, which was prepared by the National Institute for Clinical Excellence in 2006, it is recommended that energy intake should be slow and low so as to increase the weight in the range of $0.5-1 \mathrm{~kg} /$ week (13). However, the Guidelines for Treatment of Eating Disorders by the Psychiatric Association of Australia and New Zealand pointed out conflicting and insufficient findings in the literature in 2014 and recommended that the middle way should be followed. For patients with AN, it is recommended to start with $1,400 \mathrm{kcal} /$ day and increase by $450 \mathrm{kcal}$ every 3 days until the person reaches a sufficient weight. It was also stated that in addition to energy intake, patients with a risk of RFS should be given prophylactic phosphate and thiamine during the first 
7-10 days, which should be continued based on the clinical course. It is also emphasized that starting to feed patients with a high risk of RFS by the nasogastric way and with a low carbohydrate content $(40 \%-50 \%)$ is important in terms of preventing post-meal reactive hypoglycemia that develops due to insulin release and inadequate glucose stores (14).

\section{Research Effects}

Although RFS is preventable, it is a serious clinical problem that may be fatal if unnoticed. Therefore, it is important to increase the awareness of clinicians regarding the importance of early diagnosis and its management. Studies on the content and speed of refeeding offer different suggestions; therefore, there is a need for randomized controlled studies that can guide in this regard. In addition, many of the studies have been performed in adults, and studies with different age groups will contribute to a better understanding of this syndrome.

\section{Conclusion}

As a result, the most important prevention or conservation method is awareness about RFS and adequate evaluation, planning, and follow-up of patients at risk. Support with primarily prophylactic PO4, thiamine, vitamins, minerals, and energy supply at medium speed will be preventive. Of course, the refeeding protocol should be individualized according to each patient's clinical situation and planned in collaboration with experts from the relevant fields.

Peer-review: Externally peer-reviewed.

Author Contributions: Concept - E.K., A.G.; Design - E.K., A.G.; Supervision - E.K., A.G.; Literature Review - E.K., A.G.; Writing - E.K., A.G.; Critical Review - E.K., A.G.; Other - E.K., A.G.

Conflict of Interest: No conflict of interest was declared by the authors.

Financial Disclosure: The authors declared that this study has received no financial support.

\section{References}

1. Crook MA, Hally V, Panteli JV. The importance of the refeeding syndrome. Nutrition 2001; 17: 632-7. [CrossRef]

2. Mehanna HM, Moledina J, Travis J. Refeeding syndrome: what it is, and how to prevent and treat it. BMJ: British Medical Journal 2008; 336: 1495. [CrossRef]

3. O'Connor G and Nicholls D. Refeeding Hypophosphatemia in Adolescents With Anorexia Nervosa A Systematic Review. Nutr Clin Pract 2013; 28: 358-64. [CrossRef]

4. Khan LU, Ahmed J, Khan S, MacFie J. Refeeding syndrome: a literature review. Gastroenterol Res Pract 2011; pii: 410971. [CrossRef]

5. Bufano G, Bellini C, Cervellin G, Coscelli C. Enteral feeding in anorexia nervosa. JPEN J Parenter Enteral Nutr 1990; 14: 404-7. [CrossRef]

6. Van Dissel JT, Gerritsen HJ, Meinders AE. Severe hypophosphatemia in a patient with anorexia nervosa during oral feeding. Mineral and Electrolyte Metabolism 1991; 18: 365-9.

7. Sobotka L. Basics in Clinical Nutrition: Refeeding syndrome. Clinical Nutrition Espen 2010; 5: e146-7. [CrossRef]

8. Rigaud D, Boulier A, Tallonneau I, Brindisi, MC, Rozen R. Body fluid retention and body weight change in anorexia nervosa patients during refeeding. Clinical Nutrition 2010; 29: 749-55. [CrossRef]

9. Panteli, JV and Crook MA. Refeeding syndrome still needs to be recognized and managed appropriately. Nutrition 2009; 25: 130-1. [CrossRef]

10. O'Connor G, Goldin J. The refeeding syndrome and glucose load. Int J Eat Disord 2011; 44: 182-5.

11. Marikar D, Reynolds S, Moghraby OS. Junior MARSIPAN (Management of Really Sick Patients with Anorexia Nervosa). Arch Dis Child Educ Pract Ed 2015; pii: edpract-2015-308679.

12. Garber AK, Michihata N, Hetnal K, Shafer MA, Moscicki AB. A prospective examination of weight gain in hospitalized adolescents with anorexia nervosa on a recommended refeeding protocol. J Adolesc Health 2012; 50: 24-9. [CrossRef]

13. Vincent RP, Omar S, Elnenaei MO, Goo CH, Salota R, Wu P, et al. Adherence to the National Institute of Clinical Excellence guidance on parenteral nutrition screening is not enough to improve outcomes. Clin Nutr 2013; 32: 73-6. [CrossRef]

14. Hay P, Chinn D, Forbes D, Madden S, Newton R, Sugenor L, et al. Royal Australian and New Zealand College of Psychiatrists. Royal Australian and New Zealand College of Psychiatrists clinical practice guidelines for the treatment of eating disorders. Aust N Z J Psychiatry 2014; 48: 977-1008. [CrossRef] 